\title{
Three dimensional modeling method of MIMICS adjacent mask spherical open cell aluminum foam- polyurethane composites based on DICOM data
}

\author{
*Ming-si Qi ${ }^{1}$, * Wei Zhang ${ }^{2}$, Guang-ming Ren², Yi-ping Yin², Fan Zhang ${ }^{3}$, Chun-Xue Lu ${ }^{3}$ \\ 1. North University of China, Taiyuan 030051, China \\ 2. Taiyuan Heating Power Groups Co., Ltd., Taiyuan 030001, China \\ 3. Baotou North Hua'an Real Estate Co., Ltd., Baotou 014030, China
}

\begin{abstract}
The complexity and irregularity of the internal structure of aluminum foam and the particularity of the fabricating method make it difficult to establish a three-dimensional model that can accurately reflect the internal structure of aluminum foam-polyurethane composites. This study presents a method of threedimensional reconstruction of the Materialise's Interactive Medical Image Control System (MIMICS) adjacent mask based on Digital Imaging and Communications in Medicine (DICOM) data. Firstly, spherical open cell aluminum foam-polyurethane composites with different pore sizes $(5 \mathrm{~mm}, 7 \mathrm{~mm}, 9 \mathrm{~mm})$ were prepared. Then the corresponding DICOM data of the spherical open cell aluminum foam-polyurethane composites were obtained by CT-scan. Based on adjacent mask, the three-dimensional simulation model of spherical open cell aluminum foam-polyurethane composites was successfully reconstructed in MIMICS. In order to verify the feasibility of this modeling method, a quasi-static compression simulation of the three-dimensional simulation model was carried out by ABAQUS, and the simulated stress-strain curves were acquired. The quasi-static compression experiments were performed on the prepared aluminum foam-polyurethane composites, and the experimental nominal stress-strain curves were obtained. The simulated nominal stress-strain curves were compared with the experimental nominal stress-strain curves. Results show that the two curves are basically the same. The feasibility and accuracy of the three-dimensional modeling method for the aluminum foampolyurethane composites are verified.
\end{abstract}

Key words: aluminum foam-polyurethane; CT-scan; quasi-static compression; 3D modeling; simulation

CLC numbers: TG146.21 Document code: A Article ID: 1672-6421(2020)04-314-06

$\mathrm{T}$

he aluminum foam-polyurethane composite is a

kind of functional composite with complex internal structures, and its mechanical properties greatly depend on its internal structure. With the rapid development of computer technology ${ }^{[1-4]}$, computer simulation technology has become a very important means to study aluminum foam-polyurethane composites. As a complex structural composite material, aluminum foam matrix has the characteristics of complex internal cavity and irregular reproducibility. Therefore, it is difficult to establish a three-dimensional model that can accurately

\section{*Ming-si Qi}

Male, Ph.D., Associate Professor. His research interests mainly focus on buffer protection technology, intelligent medical appliances and intelligent medical robot.

E-mail: 491412397@qq.com

**Wei Zhang

E-mail: 503655163@qq.com

Received: 2019-08-28; Accepted: 2020-03-12

reflect the internal structure of aluminum foampolyurethane composites.

As early as 1982, Gibson and Ashby ${ }^{[5]}$ established the hexahedral open-cell and closed-cell foam models based on a single-hole model to simulate foam materials, and obtained some basic properties of metal foam materials by simulation, such as elasticity modulus, yield strength and other mechanical properties. Because it was a simple model, it could only be used for the foamed structure material with a relative lower density. After correcting and optimizing this simple model, the cubic plate model was developed, and then the docecahedral ${ }^{[6]}$ and tetrakaidecahedral models ${ }^{[7]}$ were generated through further model optimization. However, these simple models could not approximate the real inner pore structure of metal foam, leading to significant difference between the simulation results and the experimental results.

Subsequently, the Voronoi model was applied to the three-dimensional modeling of foamed metal materials ${ }^{[8]}$, and aluminum foam models have since been established 
by several scholars. Zhang et al. ${ }^{[9]}$ and Tang et al. ${ }^{[10]}$ studied the yield properties of aluminum foam under the triaxial strength test, and verified the veracity and scientificity of the finite element model through a uniaxial compression test with optimized modeling and calculated parameters. Nie et al. ${ }^{[11]}$ had established a 3D model of the aluminum foam by Laguerre-Voronoi and the compression performance was studied by ANSYS. The numerical results agreed very well with the test results. The Voronoi model based on Voronoi theory is set with the element of equal wall thickness. It is commonly used to simulate a closed cell aluminum foam with irregular pore shape, but in this current study, the spherical open-cell aluminum foam with regular inner structure was adopted, therefore this method was not suitable.

With the rapid development of X-ray computed tomography technology, CT scanning is the most effective way to reconstruct the structure of foam metal materials ${ }^{[12-17]}$. On this basis, this study presents a method of three-dimensional reconstruction of MIMICS adjacent mask based on DICOM data. Firstly, the spherical open cell aluminum foampolyurethane composite was scanned by CT to get DICOM data, and then the three-dimensional simulation of matrix spherical open cell aluminum foam was reconstructed in
MIMICS. Secondly, the polyurethane model was reconstructed by Boolean algorithm based on the adjacent mask on the basis of spherical open cell aluminum foam mask. Finally, the threedimensional model of spherical open cell aluminum foampolyurethane composites was successfully reconstructed. To verify the feasibility of this modeling method, the quasi-static compression simulation was carried out by ABAQUS, then the simulated stress-strain curves were obtained and compared with the experimental curves for further analysis.

\section{Specimen preparation}

Spherical open-cell aluminum foams with three different pore sizes, marked as \#1, \#2 and \#3 foams, were produced using the infiltration casting method by Beijing Zhongshi Qiangye Foam Metal Co., Ltd. The average pore diameters of the three kinds of aluminum foams are $5 \mathrm{~mm}, 7 \mathrm{~mm}$ and $9 \mathrm{~mm}$, and the corresponding average diameters of the wall pores are $1.5 \mathrm{~mm}$, $2.5 \mathrm{~mm}$ and $4 \mathrm{~mm}$, respectively. Three kinds of cylindrical specimens with size of $\Phi 35 \mathrm{~mm} \times 20 \mathrm{~mm}$ were prepared, as shown in Fig. 1. The aluminum foam specimens were cleaned and then dried in a constant temperature blast dryer.

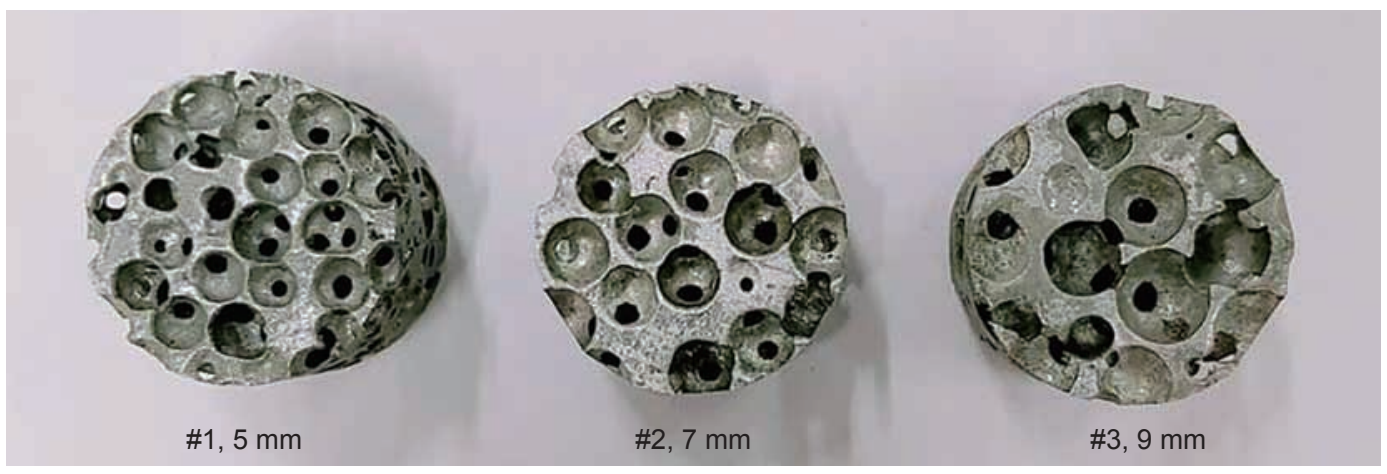

Fig. 1: Aluminum foam specimens with different pore sizes

The 9370A polyurethane particles with Shore A hardness of 70 were used for the preparation of the aluminum foam- polyurethane composite material with the following procedures: placing the weighted polyurethane material into a dry three mouth open reactor with the capacity of $1,000 \mathrm{ml}$, heating the loaded material with $220^{\circ} \mathrm{C}$ oil bath, stirring with a motorized agitator at the speed of $200 \mathrm{rpm}$ until the polyurethane material is completely fused; then prepouring the fused polyurethane liquid into the bottom of a cylindrical mold, with the size of $\Phi 36 \mathrm{~mm} \times 30 \mathrm{~mm}$, made of polytetrafluoroethylene (PTFE); pressing the aluminum foam specimen into the mold manually and making it to be the center position, at this time the polyurethane liquid infiltrates into the cell pores of the aluminum foam; continually pouring the polyurethane liquid into the mold until it covers $2-3 \mathrm{~mm}$ of the upper surface of the aluminum foam specimen; closing the end cover of the mold and pressing down the aluminum foam repeatedly to force the polyurethane liquid into the cell pores as much as possible; putting the mold into the $100{ }^{\circ} \mathrm{C}$ air drying oven to cure for $12 \mathrm{~h}$, and then taking out the spherical open cell aluminum foam-polyurethane composite specimen. The prepared specimen was polished with a BOSCH-GBG8 grinding machine until the surface was smooth. The aluminum foam-polyurethane composite specimens are shown in Fig. 2. At last, the prepared aluminum foam-polyurethane specimens were scanned using the Philips spiral CT machine with 64 rows and 128 layers, to get the corresponding scanning data DICOM files.
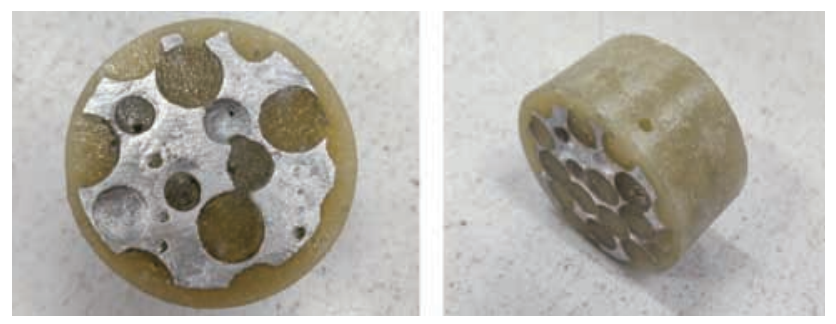

Fig. 2: Specimens of spherical open-cell aluminum foam-polyurethane composite 


\section{3D modeling for aluminum foam- polyurethane based on MIMICS}

Taking the \#2 specimen as an example, the scanning data of spherical open-cell aluminum foam were transferred to finite element model through the following steps:

Step 1: Importing the DICOM document of \#2 specimen into the MIMICS software.

Step 2: Filteringby using the Binomial blur module in the MMICICS software to reduce the influence of image noise and improve the image quality.

Step 3: Building a 3D model by firstly, reconstructing the 3D model of aluminum foam as shown in Fig. 3, then reconstructing a cylindrical solid 3D model of polyurethane using the Boolean operation, and at last, combining the $3 \mathrm{D}$ model for aluminum foam with the polyurethane 3D model, as shown in Fig. 4.

Step 4: Conducting treatment before finite element simulation (FEA module treatment) and non-mainstream meshing.

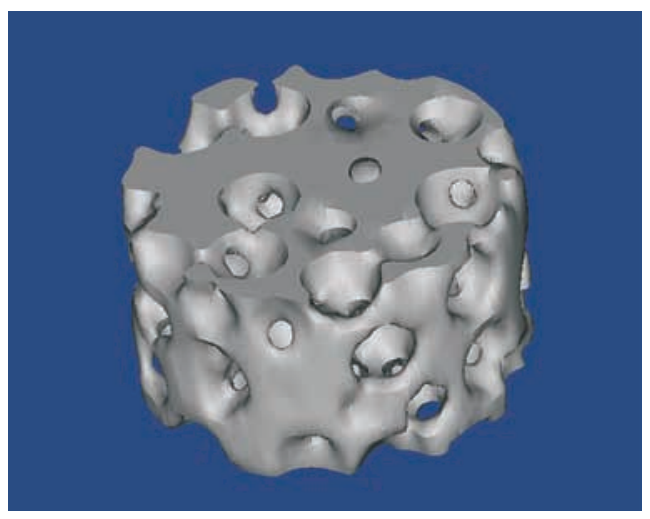

Fig. 3: 3D model of \#2 inner aluminum foam
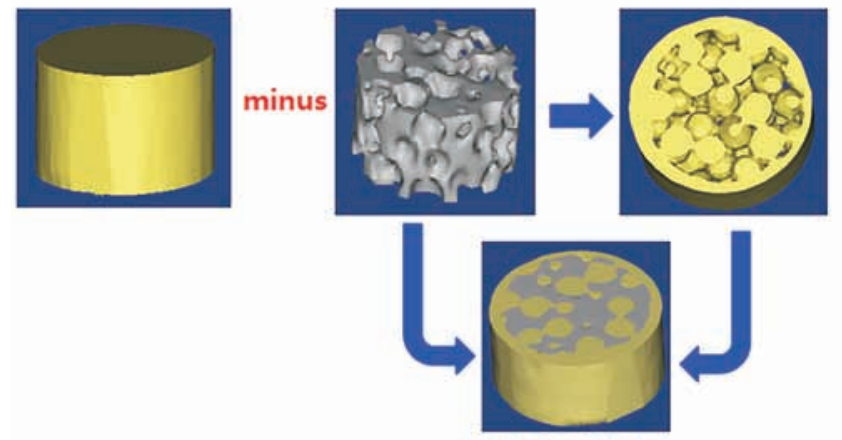

Aluminum foam

Fig. 4: 3D-modeling for aluminum foam-polyurethane composites (Boolean operation)

The two materials combined within the composite material can be divided based on masking calculation model mesh in MIMICS software.

Achieving this requires first performing a masking calculation based on polyurethane 3D model which is reverse designed after Boolean operation, to obtain the mask of polyurethane through reverse calculation by using built model, to combine with the mask of aluminum foam, to use Create Voxel Mesh in FEA module to combine the aluminum foam masking with polyurethane masking together, and then to perform optimization by the following steps:

(a) Filtering the acuminate triangular patch.

Select Fix module in 3-matic interface and enter, then select Filter Sharp Triangles operation, set filtration parameter and then filter the acuminate triangular patch in the model.

(b) Fairing the triangular patch of the 3D model surface of aluminum foam-polyurethane composite material.

Select the Fix module in 3-matic interface and enter, then select Smooth operation, set fairing parameters, and then fair the triangular patch of 3D model surface of the aluminum foam-polyurethane composite material.

(c) Reducing the triangular meshes quantity in 3D model of the aluminum foam-polyurethane composite material.

Select the Fix module in 3-matic interface and enter it, then select Reduce operation, set the mesh size parameter needed to be deleted and then reduce the triangular mesh quantity in the model.

(d) Filtering tiny meshes.

Select Fix module in 3-matic interface and enter, then select Filter Small Edges operation, filter and delete the meshes which are lower than the average value $(1 \mathrm{~mm})$.

(e) Automatically optimizing the triangular meshes.

Select Remesh module in 3-matic interface and enter, then select Auto Remesh operation, set parameter optimization and then give automatic optimization for the triangular meshes of 3D model of the aluminum foam-polyurethane composite material.

(f) Meshing the 3D model of the aluminum foam-polyurethane composite material.

Select the Remech module in 3-matic interface and enter, then select Create Volume Mesh operation, build 3D meshes.

(g) Checking the mesh quality for 3D model of the aluminum foam-polyurethane composite material.

Select Remesh module in 3-matic interface and enter, select Analyze Mesh Quality operation, check built 3D meshes quality, and if the meshes quality does not meet the requirements of the finite element simulation, analyze the reasons, then optimize meshes again until it reaches the requirements; if the meshes quality reaches the requirements, quit the mesh optimization, and then export the corresponding files. The optimized finite element model of the aluminum foam-polyurethane composite material is shown in Fig. 5.

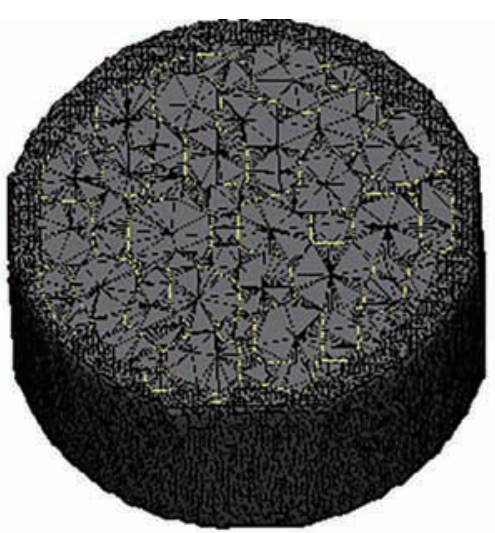

Fig. 5: 3D model mesh for \#2 aluminum foam-polyurethane composite material 
Step 5: Setting the material parameters of the model.

After finishing the 3D model meshing of the aluminum foam-polyurethane composite material, import the file into MIMICS, select FEA module and enter, select Material operation, and then enter Material Assignment interface. Give the material attribute for the meshed finite element model, and set the parameters for the model material.

\section{Quasi-static compression simulation and experiment}

For importing the built cylindrical spherical open-cell aluminum foam-polyurethane model into ABAQUS/CAE, the square discrete rigid plates with the size of $60 \mathrm{~mm} \times 60 \mathrm{~mm}$ are used for the upper and lower surface of the model to be loaded. Reference points are set at the center of these two plates respectively named as Upper and Lower, thus the motion of the reference points can be used to represent the motion of the rigid bodies. The built finite element model of spherical open-cell aluminum foam-polyurethane is given in Fig. 6.

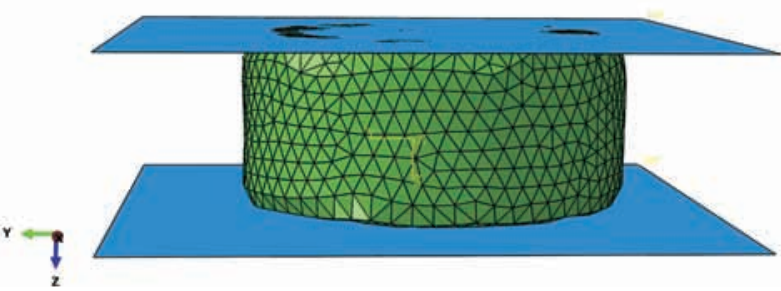

Fig. 6: Quasi-static compression finite element model for spherical open-cell aluminum foam-polyurethane

The ABAQUS/Standard analysis module was used to give the finite element analysis and solve for the quasi-static compression process of the aluminum foam-polyurethane. The material property in ABAQUS refers in particular to the cross sectional features including material property. By defining the reliable parameter for the material and cross section, the material can be presented the similar properties comparing to the actual mechanical characteristics. In the present study, the mass density for the 1,100 series of aluminum is set to be $2.7 \mathrm{e}-9 \mathrm{t} \cdot \mathrm{mm}^{-3}$, elastic modulus 70,000 MPa, Poisson's ratio 0.31 , and yield strength 80 $\mathrm{MPa}$. Polyurethane is a kind of super elastic material.

The Von-Mises Stress cloud picture for specimens of the spherical open-cell aluminum foam-polyurethane composite material is shown in Fig. 7 (in order to observe the deformation and stress situation for two materials conveniently, the upper and lower plates are hidden). The quasi-static compression load-displacement curves for each specimen are given in Fig. 8.

As known in Fig. 7, when the bearing load of the aluminum foam-polyurethane composite material changes, the skeleton of the aluminum foam starts to be loaded at the elastic stage [Fig. 7(a)], and the simulation analysis meets the characteristics at this stage. Then, with increasing bearing load, the polyurethane starts to be loaded. As shown in Figs. 7(b) and (c), the simulation analysis is in accordance with the basic characteristics of the quasi-static compression of the aluminum foam-polyurethane.
Thus, the modeling method is indirectly proved to be effective for the quasi-static compression of the composite material ${ }^{[18]}$.
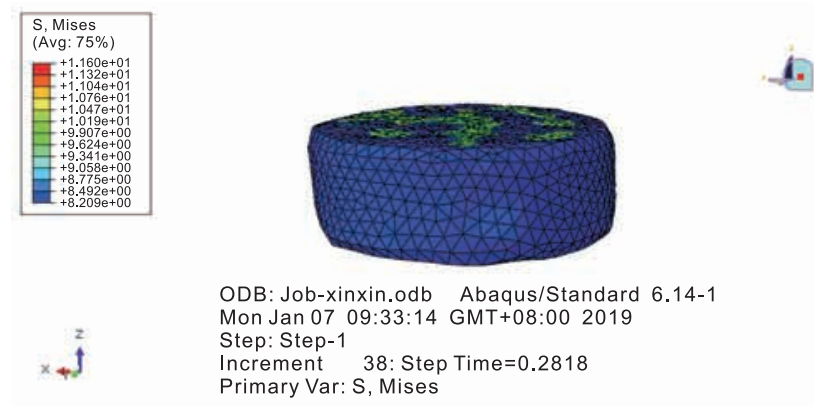

(a) Von-Mises stress cloud picture for \#3 specimen at $0.2818 \mathrm{~s}$
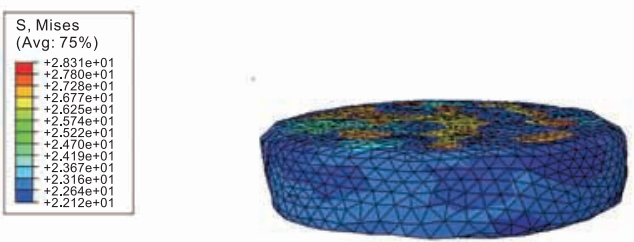

ODB: Job-xinxin.odb Abaqus/Standard 6.14-1 Mon Jan 07 09:33:14 GMT+08:00 2019 Step: Step-1 $\begin{array}{ll}z & \text { Increment } \quad 49: \text { Step Time }=0.7130 \\ & \text { Primary Var: S, Mises }\end{array}$

(b) Von-Mises stress cloud picture for \#3 specimen at $0.7130 \mathrm{~s}$
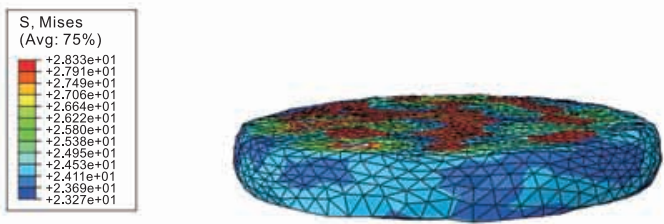

ODB: Job-xinxin.odb Abaqus/Standard 6.14-1 Mon Jan 07 09:33:14 GMT+08:00 2019

Step: Step-1

$2 \quad$ Increment 65: Step Time $=0.9991$ $x+\frac{1}{1} \quad$ Primary Var: $\mathrm{S}$, Mises

(c) Von-Mises stress cloud picture for \#3 specimen at $0.9991 \mathrm{~s}$

Fig. 7: Von-Mises stress cloud picture for \#3 specimen of aluminum foam-polyurethane

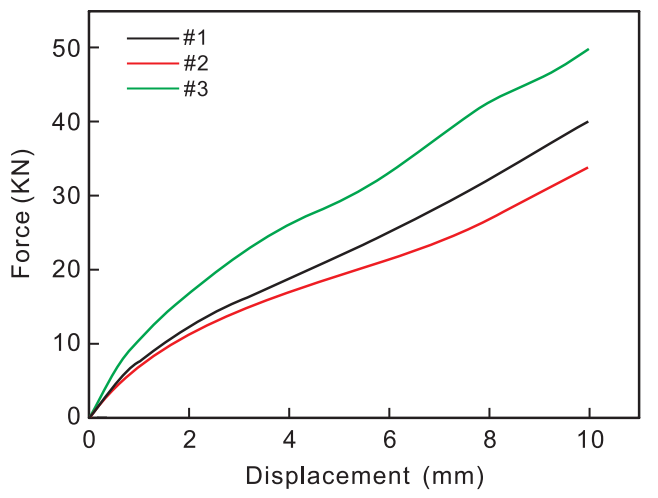

Fig. 8: Load-displacement simulation curves for \#1, \#2, \#3 specimens of aluminum foam-polyurethane

In this study, the micro-computer controlled spring testing machine (LS-W50000A) was used to implement the quasistatic compression test on the spherical open-cell aluminum foam-polyurethane composite material, as shown in Fig. 9. The maximum load for the machine is $50,000 \mathrm{~N}$, and the 
loading rate was set to $5 \mathrm{~mm} \cdot \mathrm{min}^{-1}$. The corresponding loaddisplacement curve was obtained by compression test, which is plugged into Eqs. (1) and (2) to obtain the corresponding nominal strain-stress curve. Then using Origin software to plot, the strain-stress curves can be obtained respectively for $\# 1$, \#2 and \#3 specimens (shown in Fig. 10).

$$
\begin{gathered}
\sigma_{\mathrm{n}}=\frac{F}{A_{0}} \\
\varepsilon_{\mathrm{n}}=\frac{\Delta l}{l_{0}}
\end{gathered}
$$

where, $\sigma_{\mathrm{n}}$ represents the material nominal stress, $F$ the bearing load, $A_{0}$ the original sectional area of the specimen (before compression), $\varepsilon_{\mathrm{n}}$ the material nominal strain, $\Delta l$ the thickness variation of the compressed specimen, and $l_{0}$ the original thickness of the specimen (before compression).

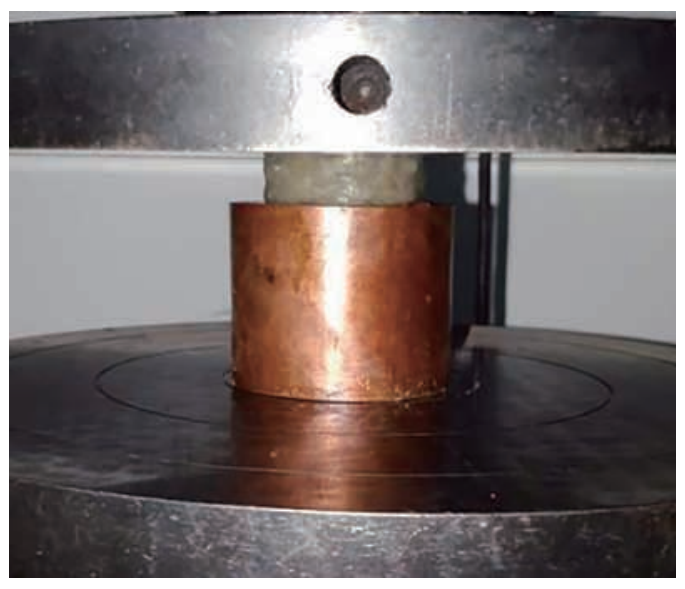

Fig. 9: Quasi-static compression test process

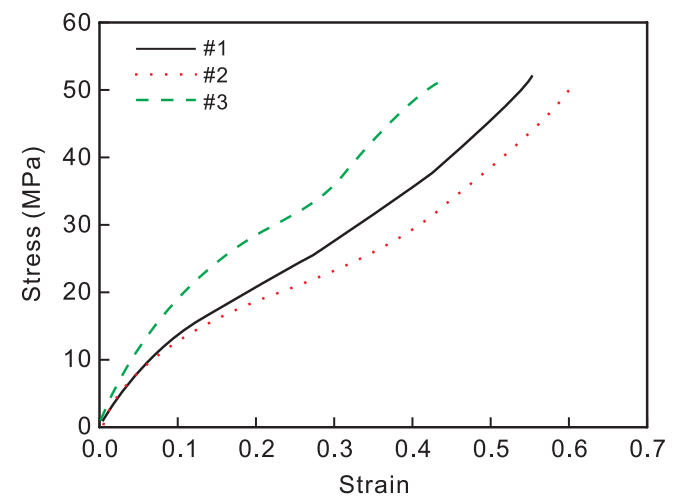

Fig. 10: Strain-stress curves of aluminum foampolyurethane composite materials

\section{Comparison for stress-strain curves between simulation and test}

The comparisons of the stress-strain curves for specimens $\# 1, \# 2$ and \#3 of the aluminum foam-polyurethane between simulation results and test results are given in Figs. 11, 12 and 13 , respectively.

It can be seen that the test results and simulation results

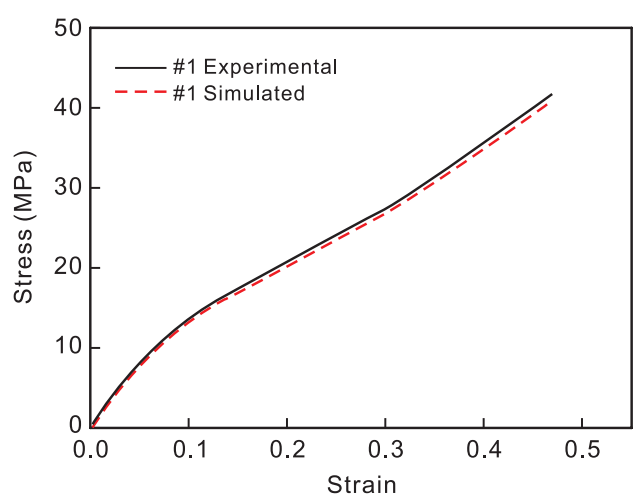

Fig. 11: Stress-strain comparison curves for \#1 aluminum foam-polyurethane specimen between simulation and test

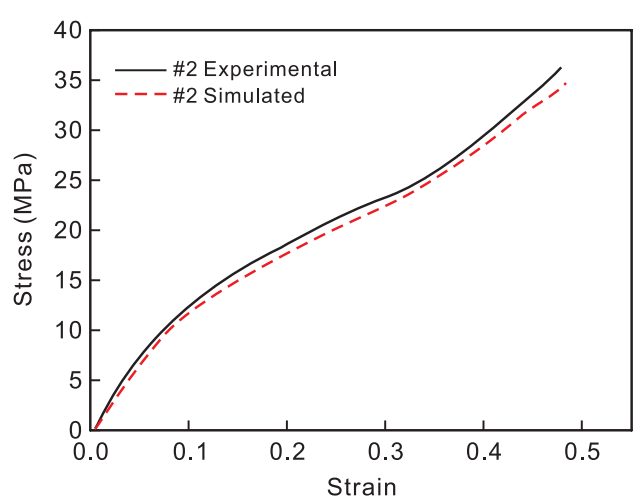

Fig. 12: Stress-strain comparison curves for \#2 aluminum foam-polyurethane specimen between simulation and test

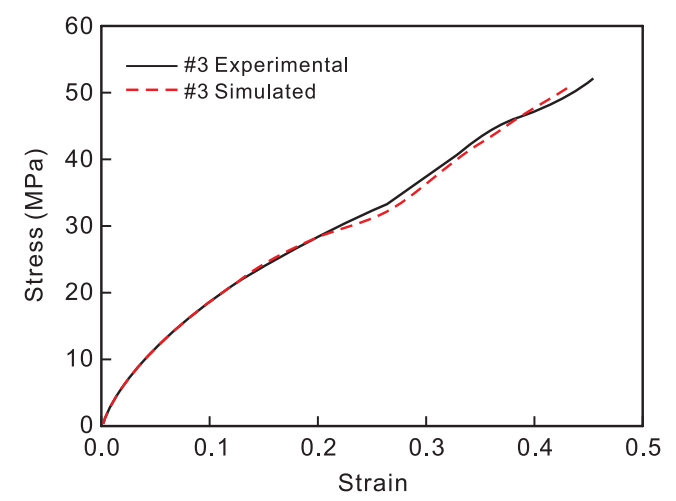

Fig. 13: Stress-strain comparison curves for \#3 aluminum foam-polyurethane specimen between simulation and test

for the strain-stress curves of these three composite material specimens are well matched in general. The test results and simulation results at elastic stage are anastomose, but have errors at the plastic stage, mainly caused by the following points. Firstly, although the built 3D model of the aluminum foam has structure very close to the real aluminum foam structure, there is still a little difference. Secondly, when preparing the aluminum foam-polyurethane composite material, part of the inner pores may not be fully filled, which are however considered as $100 \%$ filling based on Boolean algorithm to build a 3D model. Thirdly, during simulation, 
the contact surface to the model by the upper and lower plates may have some deviation with the real situation, causing some errors in results. Thus, there are some deviations to the real inner structure of the specimen, resulting in errors between simulation results and test results.

\section{Conclusion}

Aiming at the spherical open cell aluminum foam-polyurethane composites, this paper presents a method of three-dimensional reconstruction of MIMICS adjacent mask based on DICOM data. For the reconstructed spherical open-cell aluminum foam-polyurethane composites, the quasi-static compression simulation was carried out by Version 6.14 ABAQUS software. It was proved that the simulated process was in line with the quasi-static compression property of the spherical open-cell aluminum foam-polyurethane composites. The simulated stress-strain curves agreed well with the quasistatic compression property of the composites. Comparing the nominal stress-strain curves obtained from the simulation with those from experiments, the two sets of curves were basically the same. It proved that this modeling method was effective for the quasi-static compression based on the aluminum foampolyurethane composites.

\section{References}

[1] Wang Z J, Zhou J, Wang J Y, et al. A novel fault diagnosis method of gearbox based on maximum kurtosis spectral entropy deconvolution. IEEE Access, 2019, 7(1): 29520-29532.

[2] Wang Z J, Du W H, Wang J Y, et al. Research and application of improved adaptive MOMEDA fault diagnosis method. Measurement, 2019,140: 63-75.

[3] Wang Z J, Wang J Y, Du W H. Research on fault diagnosis of gearbox with improved variational mode decomposition. Sensors, 2018, 18(10): 3510, doi: 10.3390/s18103510.

[4] Wang Z J, He G F, Du W H, et al. Application of parameter optimized variational mode decomposition method in fault diagnosis of gearbox. IEEE Access, 2019, 7: 44871-44882.

[5] Gibson I J, Ashby M F. The mechanics of three-dimensional cellular materials. Proceedings of the Royal Society of London. Series A: Mathematical Physical \& Engineering Sciences, 1982, 382(1782): 43-59.
[6] Hedayati R, Sadighi M, Mohammadi-Aghdam M, et al. Comparison of elastic properties of open-cell metallic biomaterials with different unit cell types. Journal of Biomedical Materials Research Part B: Applied Biomaterials, 2017, 106 (1): 386-398.

[7] Li K, Gao X L, Roy A K. Micromechanics model for threedimensional open-cell foams using a tetrakai decahedral unit cell and Castiglianol"s second theorem. Composites Science and Technology, 2003, 63(12): 1769-1781.

[8] Wang $H$, Liu B, Kang $Y X$, et al. Analysing effective thermal conductivity of 2D closed-cell foam based on shrunk Voronoi tessellations. Archives of Mechanics, 2017, 69 (6): 451-470.

[9] Zhang X Y, Tang L Q, Liu Z J, et al. Yield properties of closedcell aluminum foam under triaxial loadings by a $3 \mathrm{D}$ Voronoi model. Mechanics of Materials, 2017, 104: 73-84.

[10] Tang $L Q$, Shi X P, Zhang L, et al. Effects of statistics of cell's size and shape irregularity on mechanical properties of $2 \mathrm{D}$ and 3D Voronoi foams. Acta Mechanica. 2014, 225: 1361-1372.

[11] Nie $Z$ W, Lin $Y Y$, Tong Q B. Modeling structures of open cell foams. Computational Materials Science, 2017, 131: 160169.

[12] Nasrabadi A A M, Hedayati R, Sadighi M. Numerical and experimental study of the mechanical response of aluminum foams under compressive loading using CT data. Journal of Theoretical \& Applied Mechanics, 2016, 54(4): 1357-1368.

[13] Ranut P, Nobile E, Mancini L. High resolution microtomographybased CFD simulation of flow and heat transfer in aluminum metal foams. Applied Thermal Engineering, 2014, 69(1-2): 230-240.

[14] Zhu X L, Ai S G, Lu X F, et al. Thermal conductivity of closed-cell aluminum foam based on the $3 \mathrm{D}$ geometrical reconstruction. International Journal of Heat \& Mass Transfer, 2014, 72: 242-249.

[15] Hangai Y, Yamaguchi R, Takahashi S, et al. Deformation behavior estimation of aluminum foam by $\mathrm{X}$-ray CT imagebased finite element analysis. Metallurgical \& Materials Transactions A, 2013, 44: 1880-1886.

[16] Jeon I, Asahina T, Kang K J, et al. Finite element simulation of the plastic collapse of closed-cell aluminum foams with X-ray computed tomography. Mechanics of Materials, 2010, 42(3): 227-236.

[17] Petit C, Maire E, Meille S, et al. Two-scale study of the fracture of an aluminum foam by X-ray tomography and finite element modeling. Materials \& Design, 2017, 120: 117-127.

[18] Qi M S, Zhang W, Wang J Y, et al. A three dimensional modeling method for spherical open cell aluminum foams based on spherical core stratification algorithm. China Foundry, 2019, 16 (4): 248-255. 